\title{
CREENCIAS Y PRÁCTICAS DE LAS MADRES RELACIONADAS CON LOS SIGNOS Y SÍNTOMAS EN LA ERUPCIÓN DENTAL DE SUS HIJOS|AS
}

\author{
${ }^{1}$ Claudia Milena Riveros Alejo, ${ }^{2}$ Laura Milena Díaz Ardila, ${ }^{2}$ Polaina Loricse Franco Díaz, ${ }^{2}$ Joyce Smith Sanabria Flórez. \\ Odontóloga Universidad El Bosque, Especialista en Gerencia Hospitalaria Escuela Superior de Administración Pública, Especialista en Relaciones Laborales \\ U. Autónoma de Bucaramanga, Especialista en Odontopediatría Universidad Santo Tomás, Docente Universidad Santo Tomás, Bucaramanga, Colombia. \\ ${ }^{2}$ Estudiante X semestre F. de Odontología Universidad Santo Tomás, Bucaramanga, Colombia.
}

Autor responsable de correspondencia: Claudia Milena Riveros A. Correo electrónico: milevale_odontopediatria@yahoo.com

RESUMEN:

Objetivo: Establecer las creencias de las madres de niños/as lactantes en relación con el proceso de erupción dental e identificar las manifestaciones sistémicas que ellas asocian como normales en dicho proceso.

Materiales y métodos: Se realizó un estudio de corte transversal. Se tomó una muestra de 87 madres de niños/as lactantes que hacían parte de la sala-cuna, caminadores y preescolar del Centro Infantil Biberones de Bucaramanga. Los datos fueron recogidos por medio de un cuestionario aplicado a las madres. Para su análisis se utilizaron las pruebas estadísticas $\mathrm{Chi}^{2} \mathrm{y}$ la $\mathrm{t}$ de $\mathrm{Student}$ $(\alpha<0.05)$.

Resultados: El 39\% de las madres asociaba la erupción con manifestaciones clínicas en el niño. Al describir los aspectos relacionados con signos y síntomas, se observó que el aumento del deseo de morder fue el más frecuente (75.9\%). Igualmente, se estableció que la conducta más utilizada por las madres para aliviar los síntomas de sus hijos es colocarles el chupo (78.2\%). Conclusiones: El estudio permitió establecer los conocimientos y las prácticas consolidadas basadas en las referencias bibliográficas existentes y así ser relacionadas con información reportada por las madres [Riveros CM, Díaz LM, Franco PL, Sanabria JS. Creencias y prácticas de las madres de niños(as) lactantes relacionadas con los signos y síntomas presentes en la erupción dental. Ustasalud 2010; $9: 96$ - 100]

Palabras clave: Creencias, Prácticas, Erupción dental.

\section{BELIEFS AND PRACTICES OF MOTHERS RELATED TO SIGNS AND SYMPTOMS ASSOCIATED WITH INFANTS TEETHING}

\section{ABSTRACT}

Objective: To establish the beliefs of mothers of children related to the process of tooth eruption and to identify associated systemic manifestations as normal to them in this process.

Methods: A cross-sectional study was done. A sample was conformed by 87 mothers of infants who were part of the nursery, toddlers and preschool Centro Infantil Biberones of Bucaramanga. Data were collected through a questionnaire administered to these mothers. For the analysis, statistical tests $\mathrm{Chi}^{2}$ and Student's t test were done $(\alpha<0.05)$.

Results: Thirty nine percent of the mothers associated teething with clinical manifestations. It was observed that increasing the desire to bite was the most frequent (75.9\%). Similarly, it was established that the behavior most often used by mothers to relieve the symptoms of their children is to put the pacifier $(78.2 \%)$.

Conclusions: This study allowed us to establish the consolidated knowledge and practices based on existing references and related information reported by mothers.

Key Words: Beliefs, Practices, Teething.

Recibido para publicación: 30 de julio de 2010. Aceptado para publicación: 10 de septiembre de 2010 . 


\section{INTRODUCCIÓN}

En la actualidad existe controversia entre madres y médicos debido a que éstos niegan la participación etiológica de la erupción de los dientes deciduos en la presencia de infecciones. ${ }^{1,2}$ El principal problema radica en la persistencia de mitos alrededor de la erupción de los dientes primarios que puedan llevar a ignorar una enfermedad real que podría poner en peligro la vida del niño. ${ }^{3}$

Los mitos alrededor del proceso de la dentición han sido muchos y de ser una entidad eminentemente patológica tal como se consideraba en el siglo XIX cuando se creía que causaba el más alto grado de mortalidad infantil se ha cambiado a una concesión fisiológica de la que se infiere la necesidad de tratar al paciente en el alivio de sus síntomas. ${ }^{4}$

De acuerdo con lo anterior, es necesario conocer las creencias y las prácticas de las madres de los lactantes en relación con el proceso de erupción. Por tal motivo, el objetivo del presente trabajo fue evaluar estas creencias y prácticas en un grupo de madres de niños(as) lactantes que asistían a un jardín infantil de la ciudad de Bucaramanga.

\section{MATERIALES Y MÉTODOS}

Se realizó un estudio de corte transversal en el que se tomó como universo a las madres que llevaban sus hijos(as) al Centro Infantil Biberones de Bucaramanga. La muestra estuvo constituida por 87 madres de los niños(as) lactantes que hacían parte de la sala-cuna, caminadores y preescolar.

Se realizó un muestreo no probabilístico por conveniencia. Entre los criterios de inclusión se consideraron a la madres que tuvieran sus hijos(as) en la sala cuna, caminadores o preescolar con edades entre los seis meses y los tres años de edad, que hubiesen recibido la encuesta y la hubiesen devuelto diligenciada a la institución. Se excluyó a las madres cuyos hijos(as) presentaran alteraciones sistémicas o estuvieran enfermos en el momento de realizar la encuesta.

Las variables que se tomaron en cuenta en el estudio fueron el sexo del niño, la edad de la madre, la edad del hijo, la condición socioeconómica, el nivel educativo de las madres, la relación entre la erupción dental y la aparición de las enfermedades, fiebre, diarrea, deseo de morder por parte de los lactantes, inflamación y enrojecimiento de la encía, aumento de salivación, apetito reducido, e irritabilidad, entre otras.
Inicialmente, se solicitó permiso en el jardín infantil para entrevistar a las madres. El cuestionario fue entregado a los docentes quienes se encargaron, a través de los niños, de hacerlos llegar a las madres de familia. Una vez las encuestas fueron diligenciadas, las madres las regresaban a la institución que las compiló y entregó a las investigadoras.

\section{Análisis estadístico}

La codificación se hizo por duplicado en Excel y Epi - Info 6.04. El análisis fue realizado en Stata 9.0. En el análisis univariado se clasificaron las variables, para las variables cualitativas se obtuvieron proporciones y razones mientras que para las cuantitativas se obtuvieron medidas de tendencia central y de dispersión..$^{5-7}$

Para el análisis bivariado se cruzaron las variables elegidas para el estudio: las variables independientes con cada una de las dependientes y variables cuantitativas con variables cualitativas para lo cual se utilizó la prueba $\mathrm{Chi}^{2}$ y la t de Student con un valor $\mathrm{p}<0.05$.

\section{Consideraciones éticas}

Para el diligenciamiento de las encuestas y la ejecución de esta investigación se siguieron las normas establecidas en la Resolución 008430 de 1993 del Ministerio de Salud de la República de Colombia para los trabajos de investigación que involucran comunidades.

\section{RESULTADOS}

Se evaluaron 87 madres de infantes, cuya edad promedio fue de 31 años, el $78.2 \%$ (68) tenía un nivel educativo superior, el $74.7 \%$ (65) trabajaban fuera de casa. El 55.2\% (48) permanecía la mayor parte del tiempo con su hijo. La edad promedio de sus hijos fué de 26 meses. El 50.6\% (44) de los infantes pertenecían al sexo femenino y el 24\% (21) eran de estrato 6 (Tabla1).

$\mathrm{Al}$ describir los aspectos relacionados con signos y síntomas, se observó que el 75.9\% (66) de las madres respondieron que su hijo(a) presentaba un aumento en el deseo de morder, seguido del enrojecimiento e inflamación de la encía con el $64.4 \%$ (56). El aumento de la salivación estuvo representado por el $62 \%$ (54), la irritabilidad en el niño con el 59.8\% (52) y la fiebre con el 54\% (47). Las enfermedades con menor frecuencia fueron la diarrea con el $26.4 \%$ (23), y la disminución del apetito con el 46\% (40) (Tabla 2).

Al analizar los aspectos relacionados con las conductas que realizaba la madre para aliviar 
los síntomas de sus hijos, el $78.2 \%$ (68) colocaba el chupo a sus hijos(as), el 49.4\% (43) llevaba a su hijo(a) al médico. El uso de remedios caseros estuvo representado por el $37.9 \%$ (33) de las madres. Sólo el 16\% (14) de las madres llevaban a sus hijos(as) al odontólogo (Tabla 3).

Tabla 1. Variables sociodemográficas.

\begin{tabular}{lc}
\hline \multicolumn{1}{c}{ VARIABLE } & FRECUENCIA (\%) \\
\hline Global & $87(100)$ \\
Sexo & $44(50.6)$ \\
Femenino & $43(49.4)$ \\
Masculino & \\
Estrato & $6(7)$ \\
3 & $43(49.4)$ \\
4 & $17(19.6)$ \\
5 & $21(24.1)$ \\
6 & \\
Nivel educativo madre & $2(2.3)$ \\
Secundaria & $68(78.2)$ \\
Educación superior & $17(19.6)$ \\
Otros & \\
Actividad de la madre & $3(3.45)$ \\
Estudia & $65(74.7)$ \\
Trabaja & $7(8.05)$ \\
Estudia y trabaja & $12(13.8)$ \\
Ninguna & \\
Tiempo con su hijo & $21(24.14)$ \\
Siempre & $48(55.2)$ \\
Casi siempre & $18(20.7)$ \\
Muy poco & 5
\end{tabular}

El 60.9\% (53) de las madres no relacionaron la erupción de los dientes con la aparición de signos y síntomas (Tabla 4). El 54\% (47) de las madres tuvo creencias adecuadas sobre los síntomas y signos del proceso de la erupción dental (Tabla 5).

Al analizar a las madres de acuerdo con las variables sociodemográficas, se encontraron diferencias estadísticamente significativas en no tener conocimiento acerca de los signos y síntomas de la erupción dental $(\mathrm{p}=0.005)$ y la práctica adecuada para aliviarlos $(\mathrm{p}=0.027)$. Adicionalmente, se encontraron menos conocimientos en un $61.7 \%$ (29) y prácticas en un $85 \%$ (34) en relación con las madres que trabajaban.

Al analizar los datos administrados por las madres de acuerdo con los signos y los síntomas se determinaron diferencias estadísticamente significativas en la no asociación con diarrea $(p=0.031)$, deseos de morder $(p=0.0001)$, enrojecimiento e inflamación de la encía $(p=0.0001)$, aumento de la salivación $(\mathrm{p}=0.001)$ e irritabilidad $(\mathrm{p}=0.008)$.

Al referirse a las madres que no tenían prácticas adecuadas para aliviar los signos y síntomas de sus hijos se encontraron diferencias estadísticamente significativas en la no asociación al llevar a sus hijos al odontólogo $(\mathrm{p}=0.0001)$, llevar a sus hijos al médico $(p=0.0001)$ suministrar hielo o bebidas heladas a sus hijos $(p=0.003)$.

Al hacer un análisis comparativo estadístico entre las creencias adecuadas de las madres y los conocimientos consolidados no se encontraron diferencias estadísticamente significativas ya que según los conocimientos consolidados, el $57.4 \%$ (27) de las madres tienen creencias adecuadas mientras que en las prácticas consolidadas existe una igualdad de opiniones.

Tabla 2. Signos y síntomas reportados por las madres.

\begin{tabular}{lc}
\hline \multicolumn{1}{c}{ VARIABLE } & $\begin{array}{c}\text { FRECUENCIA } \\
\text { (\%) }\end{array}$ \\
\hline Global & $87(100)$ \\
Fiebre & \\
No & $4(46)$ \\
Si & $47(54)$ \\
Diarrea & \\
No & $64(73.6)$ \\
Si & $23(26.4)$ \\
Deseo de morder & \\
No & $21(24.14)$ \\
Si & $66(75.9)$ \\
Enrojecimiento e inflamación de las & \\
encías & \\
No & \\
Si & $31(35.6)$ \\
Aumento de saliva & $56(64.4)$ \\
No & \\
Si & $33(38)$ \\
Apetito reducido & $54(62)$ \\
No & \\
Si & $47(54)$ \\
Irritabilidad & $40(46)$ \\
No & \\
Si & $35(40.2)$ \\
\hline & $52(53.8)$ \\
\hline
\end{tabular}


Tabla 3. Conductas realizadas por la madre para aliviar los signos y síntomas de sus hijos.

\begin{tabular}{lc}
\hline \multicolumn{1}{c}{ VARIABLE } & Frecuencia (\%) \\
\hline Global & $87(100)$ \\
Lleva su hijo al médico & $44(50.6)$ \\
No & $43(49.4)$ \\
Si & \\
Lleva su hijo al odontólogo & $73(83.9)$ \\
No & $14(16.1)$ \\
Si & \\
Utiliza remedios caseros & $54(62)$ \\
No & $33(37.9)$ \\
Si & \\
Hielo & $69(79.3)$ \\
No & $18(20.7)$ \\
Si & \\
Chupo o juguetes para morder & $19(21.8)$ \\
No & $68(78.2)$ \\
Si & \\
Bebidas heladas & $65(74.7)$ \\
No & $22(25.3)$ \\
Si & \\
Otros & $48(55.2)$ \\
No & $39(44.8)$ \\
Si & \\
Nada & $71(81.6)$ \\
No & $16(18.4)$ \\
Si &
\end{tabular}

Tabla 4. Aspectos relacionados con la erupción y aparición de enfermedad.

\begin{tabular}{lc}
\hline \multicolumn{1}{c}{ VARIABLE } & Frecuencia (\%) \\
\hline Global & $87(100)$ \\
Relación entre erupción y & \\
enfermedad & \\
No & $53(60.9)$ \\
Si & $34(39.1)$ \\
\hline
\end{tabular}

Tabla 5. Creencias apropiadas por la madre y su relación con los conocimientos y prácticas consolidadas.

\begin{tabular}{lc}
\hline \multicolumn{1}{c}{ VARIABLE } & Frecuencia (\%) \\
\hline Global & $87(100)$ \\
Creencias adecuadas & \\
No & $40(46)$ \\
$\mathrm{Si}$ & $47(54)$ \\
\hline
\end{tabular}

\section{DISCUSIÓN}

Los padres y médicos han debatido sobre la existencia de manifestaciones sistémicas asociadas a la erupción dentaria, ya que gran parte del conocimiento acerca de los signos y síntomas de la dentición está basado en la información que en la mayoría de los casos es atribuida a opiniones de los padres. ${ }^{8-19}$ Una búsqueda bibliográfica al respecto no resuelve la controversia, la acentúa, ya que es muy poco lo escrito al respecto; tampoco hay uniformidad de criterios. . $^{1,2,8-19}$

Algunos autores en sus investigaciones refieren que la erupción dental puede ir acompañada de signos y síntomas en el infante. Ramos y Hernández (2002) reportaron en su trabajo con 223 madres de niños en edades entre los 6 y 12 meses, que el 59.6\% señaló que sus hijos presentaron algún síntoma durante la erupción. ${ }^{19}$

La erupción primaria es una etapa fisiológica y anatómica de la infancia y como tal es raro para un niño atravesar este periodo de la dentición sin presentar ninguna manifestación clínica. Según Peretz y colaboradores de 147 niños, el $60.7 \%$ de la muestra total presentó por lo menos una manifestación clínica, se evidenció la salivación como la más frecuente $(15.2 \%){ }^{8}$

En el estudio realizado para identificar las creencias y prácticas relacionadas con la erupción dental en mujeres con hijos lactantes en el Centro Infantil Biberones de Bucaramanga, se estableció que el 39\% relacionó la erupción con manifestaciones clínicas en el niño.

Al describir los aspectos relacionados con signos y síntomas, se observó que el aumento del deseo de morder fue más común con el $75.9 \%$ (66), seguida del enrojecimiento e inflamación de la encía con el $64.4 \%$ (56), el aumento de la salivación se observó en el 62\% (54), la irritabilidad en el niño con el 59.8\% (52) y la fiebre con el $54 \%$ (47).

De manera similar, Feldens y colaboradores (2010) encontraron en un estudio de cohorte que el $73 \%$ de los niños observados presentaron síntomas relacionados con la erupción dental. Entre los síntomas más frecuentes se presentaron irritabilidad (40.5\%), fiebre $(38.9 \%)$, diarrea $(36.0 \%)$. La práctica más común fue administrar medicamentos para aliviar los síntomas sin que fueran recetados por el odontólogo. ${ }^{10}$

La diarrea no fue muy frecuente ya que se presentó sólo en el $26.4 \%$ (23) y la disminución del apetito en el 46\% (40). Igualmente, el estudio permitió establecer que las conductas más usadas por las madres para aliviar los síntomas de sus hijos eran colocar el chupo a sus hijos con el (78.2\%) seguido de llevar a su hijo al médico (49.4\%), así como el uso de remedios caseros (37.9\%). Sólo el 16\% de la madres llevaban su hijo(a) al odontólogo. El 54\% de las madres coincidieron con sus creencias al relacionarlos con los conocimientos 
adecuados y consolidados por parte de los investigadores.

Los resultados del estudio permitieron establecer diferencias estadísticamente significativas en el no tener conocimiento acerca de los signos y síntomas de la erupción dental y la práctica adecuada para aliviarlos con relación a las madres que trabajaban.

Es importante anotar que el presente estudio permitió a los autores establecer conocimientos y prácticas consolidadas basadas en las referencias bibliográficas existentes y así ser relacionadas con información reportada por las madres. Los conocimientos consolidados corresponden a las manifestaciones presentes en el niño que son enrojecimiento e inflamación de las encía, aumento en el deseo de morder, diarrea y fiebre. Las prácticas consolidadas hacen referencia a la conducta tomada por la madre para aliviar los síntomas de sus hijos los cuales son llevar el niño al odontólogo y llevar el niño al médico.

También se puede deducir que un alto porcentaje de madres reportaron por lo menos una manifestación clínica durante la erupción dentaria. Es necesario el desarrollo de un proceso de capacitación a las madres de lactantes del Centro Infantil Biberones de la cuidad de Bucaramanga, para que tengan conocimientos adecuados y puedan llevar a cabo prácticas apropiadas cuando se presente el proceso de erupción dentaria de sus hijos(as).

Para ello se requiere que dicho proceso de capacitación provea a la madre de familia los fundamentos que le permitan tener claridad sobre las manifestaciones normales producidas por la erupción dental. Concientizar a las madres sobre la necesidad de llevar a sus hijos al odontólogo o al médico cuando se presenten signos o síntomas diferentes para descartar algún proceso infeccioso. Se sugiere realizar estudios de cohorte para hacer un seguimiento adecuado y de esta forma establecer si existe o no asociación entre la aparición de signos y síntomas y la erupción dentaria.

\section{BIBLIOGRAFÍA}

1. Fogel CG. Signos y síntomas atribuidos a la erupción dentaria en los niños (primera parte). Arch Argent Pediatr 2004; 102: $35-43$.

2. Fogel CG. Signos y síntomas atribuidos a la erupción dentaria en los niños. una aproximación histórica (segunda parte). Arch Argent Pediatr 2004; 102: 185 - 189.

3. Ramírez LM, Sandoval GP, Ballesteros LE. Síntomas óticos y desórdenes temporomandibulares: pasado y presente. Revista Latinoamericana de Ortodoncia y Odontopediatría [en línea]. URL disponible en: http://www. ortodoncia.ws/publicaciones/2005/sintomas_oticos_desordenes_temporomandibulares.asp

4. Algazi-Bayley I. Ilena. Creencias populares en pediatría. Investigación a nivel hospitalario. Rev Med Uruguay 1990; 6: $23-33$.
5. Microsoft office Excel 2003. Microsoft Corporation.

6. CDS-OMS. Epi-info 6, Versión 6.04. Atlanta, (Nov 1996).

7. Stata Corp. Stata Statistical Software. Release 11.0. Collage station. TX: Stata Corporation 2009.

8. Abreu JM, Terrero J, García SM, Leyva MA. Manifestaciones sistémicas del brote dentario. Rev Cubana Estomatol 1997; 34: $67-70$.

9. Sood S, Sood M. Teething: myths and facts. J Clin Pediatr Dent 2010; 35: 9 - 13.

10. Feldens CA, Faraco IM, Ottoni AB, Feldens EG, Vitolo MR. Teething symptoms in the first year of life and associated factors: a cohort study. J Clin Pediatr Dent 2010; 34: $201-206$

11. Hulland SA, Luca JO, Wake MA, Hesketh KD. Eruption of the primary dentition in human infants: a prospective descriptive study. Pediatr Dent 2000; 22: 415 - 421.

12. Lannelly V. Teething [en línea]. URL disponible en: http:// pediatrics.about.com/od/teething/a/0107_teething.htm

13. Mackning ML, Piedmonte M, Jacobs J, Skibinski C. Symptoms associate with infant teething. Pediatrics 2000; 105: $747-752$.

14. Matey P. La erupción de las piezas dentales no asociada a la fiebre o a la diarrea. El Mundo 2000 Diciembre 16; Suplemento de Salud No. 413. URL disponible en: http:// www.elmundo.es/salud/2000/413/976779306.html

15. Peretz B, Ram D, Hermida L, Otero MM. Systemic manifestation during eruption of primary teeth in infants. $J$ Dent Child 2003; 70: 170 - 173.

16. Quesada B. La importancia de la higiene bucodental en edades tempranas. Revista MTA Pediatría 1994.

17. Quirós O, Quirós L, Quirós J. La orientación psicológica en el manejo de ciertos hábitos en odontología. Revista Latinoamericana de Ortodoncia y Odontopediatria [en línea]. URL disponible en: http://www.ortodoncia.ws/ publicaciones/2003/orientacion_psicologica_habitos_odontologia.asp

18. Espinosa MA, Anzures B. Dentición primaria infantil. Mitos y realidades. Rev Med Hosp Gen Mex 2003; 66: 43 - 47.

19. Shapira J, Beremstein G, Engelhard D, Cahan S. Cytokine levels in gingival crevicular fluid of erupting primary teeth correlated with systemic disturbances accompanying teething. Pediatr Dent 2008; 25: $441-448$.

20. Shout N. On teething symptoms. BMJ 2003; 326: 208.

21. Vernacchio L, Vezina RM, Mitchell AA, Lesko SM, Plaut AG, Acheson DW. Diarrhea in American infants and young children in the community setting: incidence, clinical presentation and microbiology. Pediatr Infect Dis J 2006; 25 : $2-7$

22. Wake M. Does a teething child need serious illness excluding? BMJ 2007; 92: 266 - 268.

23. Wake M, Hesketh $\mathrm{K}$, Lucas J. Teething and tooth eruption in infants: a cohort study. Pediatrics 2000; 106: $1374-$ 1379.

24. Wake M, Hesketh $\mathrm{K}$. Teething symptoms: cross sectional survey of five group professionals. BMJ 2002; 325: 814.

25. Wilson S, Badgett JT, Gould AR. Tooth eruption and otitis media: are they related? Pediatr Dent 1986; 8: 296 - 298.

26. Ramos IC, Hernández M. Síntomas asociados con la dentición infantil: mitos y realidades. Bol Méd Postgrado 2002; 18: $125-128$. 